\title{
A test of community assembly rules using foliar endophytes from a tropical forest canopy
}

\author{
Donald Julian ${ }^{1,}{ }^{*}$, Roy Mélanie ${ }^{1}$, Suescun Uxue ${ }^{1}$, Iribar Amaia ${ }^{1}$, Manzi Sophie ${ }^{1}$, Péllissier Léonie 2 , \\ Gaucher Philippe ${ }^{3}$, Chave Jérôme ${ }^{1,}{ }^{*}$
}

1 Université Toulouse 3 Paul Sabatier, CNRS IRD, UMR 5174 Evolution et Diversité Biologique (EDB)

118 route de NarbonneF-31062 Toulouse, France

2 School of Pharmaceutical Sciences EPGL, University of Geneva, University of Lausanne Rue Michel

Servet $1 \mathrm{CH}-1211 \mathrm{Geneva}$, Switzerland

${ }^{3}$ CNRS, Université de Guyane, UMRS LEEISA Campus IRD de Cayenne 97300, Cayenne

* Corresponding authors : Julian Donald, email address : julesdonald@gmail.com ; Jérôme Chave, jerome.chave@univ-tlse3.fr

\begin{abstract}
:
1.Community assembly theory assumes that ecological communities are spatially delimited into patches. Within these patches, coexistence results from environmental filtering, competition, and immigration. Truly delineated communities exist in laboratory studies of microbial cultures in Petri dishes, yet empirical tests conducted in continuous environments often use patches defined by convention as opposed to realised boundaries.
\end{abstract}

2. Here we perform a test of ecological community assembly rules using foliar endophyte communities from a tropical rainforest, where leaves are considered as patches for both fungal and bacterial communities. We determined the diversity of fungal and bacterial endophytes using environmental DNA sequencing of 365 top-canopy leaves, collected from 38 host trees belonging to 22 different species across a 4-hectare research plot. Three leaves were collected from three or more branches within each tree crown. We tested the effect of host tree species and their level of phylogenetic relatedness on community composition as well as the contribution of geographic distance between leaves to endophyte community diversity.

3.Endophyte diversity significantly differed across host tree species, as did community composition. Within certain endophytic orders (Xylariales, Rhizobiales) species assemblages significantly differed across host tree species, but this trend was weaker or non-existent in other orders known to contain pathogens and saprotrophs (Polyporales, Solirubrobacterales). Phylogenetically related host tree species displayed more similar endophyte communities than expected by chance, but geographically close trees did not. Consistent with the finding of host-specificity, nearby leaves tended to host more similar communities than distantly positioned ones.

4.These findings demonstrate that foliar endophytes are structured by dispersal across small spatial scales, but at the scale of the canopy they display patterns of neutral filtering, with only a small part of variation described by host tree differences. Endophyte communities thus act as a model system in evoking the rules predicted by theoretical community ecology. 
Keywords : Bacteria, Distribution, French Guiana, Fungi, Metabarcoding, Plant-pathogen interactions, Rainforest 


\section{Introduction}

90 Understanding the mechanisms which allow for the emergence and maintenance of species

91 diversity, and in particular the coexistence of competing species, is a challenge for

92 community ecology (Ricklefs 2004). Theory suggests that local species diversity results from

93 a balance between local constraints, including environmental conditions and competition, and

94 immigration from external sources through dispersal (Vandermeer 1972, Leibold 1995,

95 Hubbell 2001, Condit et al. 2002, Chase et al. 2005). Studies aiming to contrast the relative

96 weight of these mechanisms require spatially discrete communities of identifiable species at a

97 single trophic level, organized in discrete patches, and existing in large enough numbers to 98 allow for replication (Ellwood et al. 2009). Whilst such conditions are achievable for

99 microbiologists conducting laboratory-based experiments on communities grown in Petri

100 dishes (e.g. Maynard et al. 2017), these fail to represent the levels of variability experienced

101 by natural communities (Crowther et al. 2018). Field tests of community assembly rules have

102 often been conducted using trees in tropical rainforests forests, habitats characterized by high

103 levels of species diversity, with large levels of species turnover from one community to the

104 next (Hubbell 2001, Fine et al. 2004). In these studies, communities of trees are defined by

105 convention as opposed to realised boundaries, and as such inferences of structural patterns

106 may be biased, masking the true processes structuring ecological communities.

107 Foliar endophytes inhabit the intercellular layers of leaves, without provoking a

108 symptomatic response from the host plant, although certain species may be latent saprobes or

109 pathogens (de Bary 1886; Carroll 1988; Porras-Alfaro \& Bayman 2011). Within leaves,

110 multiple species coexist in spite of likely competition for the resources available. Because

111 they are constrained to live within leaves, in communities more delineated than other

112 microbial habitats such as soils, they represent a model example of a metacommunity.

113 Colonisation by endophytes is thought to occur soon after the flushing of new leaves, when

114 spores deposit, germinate and subsequently penetrate the leaf cuticle without provoking an

115 antipathogenic response (Arnold and Herre 2003, Griffin \& Carson 2015). Initial leaf

116 colonists are likely to maintain their dominance in relation to future colonists, resulting in an

117 effective host defence against pathogens and invertebrate herbivores (Herre et al. 2007; Van

118 Bael et al. 2018), albeit at a cost to the host plant in terms of nitrogen metabolism and

119 photosynthetic rate (Mejia et al. 2014, Christian et al. 2019). 
Foliar endophytes have been found in all sampled plant hosts (U'Ren et al. 2012), with

121 over 8,000 species of fungi, and 7,000 species of bacteria described as occurring only within

122 leaves according to a recent survey (Griffin \& Carson 2018). Tropical regions have shown to

123 support much higher levels of endophyte diversity than temperate regions (Arnold et al. 2000,

124 Arnold \& Engelbrecht 2007, Arnold \& Lutzoni 2007), although the factors responsible for

125 generating such high levels of diversity remain elusive, as well as the mechanisms that

126 structure communities within leaves. Studies of bacterial endophytes are for the most part

127 limited to their occurrence in agricultural crops or trees in temperate regions (Griffin \&

128 Carson 2015, Miyambo et al. 2016, Carrell \& Carper 2016). The roles of host tree species

129 (Lodge 1997, Arnold \& Herre 2003), dispersal among endophyte communities (Cordier et al.

130 2012, Izuno et al. 2016), and abiotic conditions (Suryanararanan et al. 2002, 2003,

131 Zimmerman \& Vitousek 2012) have all been explored. In these studies, only part of

132 community variation was explained, with the idea of community composition as shaped by

133 neutral processes largely ignored. Indeed, these studies have focused on leaf samples

134 collected from the forest understory, potentially masking the greater levels of wind-driven

135 dispersal in the upper canopy (Gilbert \& Reynolds 2005), and restricting the sampling to

136 leaves that contribute little to the overall net primary production of tropical forest ecosystems.

137 Furthermore, the majority of previous studies have relied on techniques which represent

138 endophyte communities based on fungi and bacteria detected following tissue culturing,

139 although it is known that many species do not grow easily on standard culture media (Hyde \&

140 Soytong 2008). Environmental DNA (eDNA) sequencing potentially captures a greater

141 breadth of the diversity associated with environmental samples by identifying of observable

142 taxonomic units (OTUs) as proxies for species, and thus is a promising technique for the

143 exploration of endophyte diversity within leaf samples (e.g. Vincent et al. 2016, Del Olmo-

144 Ruiz \& Arnold 2017, Haruna et al. 2017).

145 This study aims to elucidate the factors structuring ecological communities, by

146 characterising the diversity of upper-canopy fungal and bacterial endophytes in a primary

147 rainforest, and shifts in their community composition across phylogenetic and geographical

148 gradients. Because endophytes are restricted to leaf habitats, this system represents an ideal

149 setup to test community ecology theory. Our study makes use of an innovative canopy-access

150 system to sample upper crown leaves from multiple host trees across a fully inventoried 4-ha

151 permanent tree plot. If foliar endophyte communities are structured to a greater extent by their

152 niche, then we would expect to see a strong correlation between the composition of endophyte 
153 communities and their host plants. This is to be expected if closely allied host species are

154 more similar with respect to their foliar functional traits than unrelated species (i.e. the traits

155 are phylogenetically conserved as shown by Baraloto et al. (2012) and Courtois et al. (2016)).

156 If dispersal limitation is important in structuring communities, then we would expect to see

157 proximal communities within the canopy following an isolation-by-distance pattern, from

158 leaves sharing the same branch, to those associated with separate tree crowns across the study 159 plot.

160 We hypothesize that at the scale of the 4-ha plot, canopy endophyte assemblages will

161 display patterns of neutral structuration as a result of dispersal limitation. However, when

162 controlling for the scale at which communities assemble, we expect that host tree species will

163 determine endophyte colonization success, and that closely allied host species will match this

164 by having more similar communities than distantly related species. Similarly, we expect that

165 within crowns, proximate leaves will be more similar than distant leaves. In particular, we

166 hypothesize that the response of endophyte occurrence within certain host trees will depend

167 on their level of host specificity, detected when focusing on endophyte community responses

168 within specific fungal and bacterial orders.

\section{Materials and Methods}

170 Sample Site

171 Data collection took place at the Nouragues Ecological Research Station in French Guiana,

172 within an area of pristine lowland tropical rainforest protected since 1996. This area is

173 characterised by a high level of floral diversity, with over 1700 recorded angiosperm species

174 (Sabatier, 1990, van der Meer and Bongers, 1996). The reserve experiences mid-day

175 temperatures of $26^{\circ} \mathrm{C}$ and rainfall of $2861 \mathrm{~mm}$ per year (Réjou-Méchain et al. 2015). It

176 experiences two dry seasons, a longer dry season between September and mid-November, and

177 a shorter dry season in March.

178 Fieldwork described here took place in August 2017. Sampling was conducted in a 4-

179 ha permanent sampling plot adjacent to the Pararé research station $\left(4^{\circ} 02^{\prime} \mathrm{N}, 52^{\circ} 41^{\prime} \mathrm{W}\right)$

180 located on the Arataye tributary of the Approuague River. Access to the canopy was made

181 possible by the 'Canopy Operational Permanent Access System' (COPAS), consisting of a

182 trio of $45 \mathrm{~m}$ high pylons set up at the vertices of an equilateral triangle with edges $180 \mathrm{~m}$ in 
183 length, equipped with a mechanically operated harness which allows for the displacement of

184 an individual anywhere within the upper canopy across the research plot (Fig. 1).

185 Sample design and preparation

186 Leaves were collected from the upper crown of 38 trees, of 22 different species, selected to

187 represent a phylogenetically diverse range of species (Fig. 2 \& S.I. 1). A stratified sampling

188 design was conducted for each tree: three leaves were collected from each of a minimum of

189 three branches positioned across the crown. For those trees with a large crown, 4 or 5

190 branches were selected accordingly, so that sampling accurately represented the total spread

191 of the crown. In total this resulted in 365 leaves.

192 Leaves were immediately transported to a field laboratory where they were surface

193 sterilised in order to ensure that sampling consisted of only endophytes. This sterilisation

194 included several steps, first mechanical cleaning and scraping with toothbrushes. Secondly,

195 following from the protocol detailed by Arnold et al. (2000), leaves were soaked in diluted

196 bleach $(0,525 \%)$ for $2 \mathrm{~min}$ before transfer into ethanol $(70 \%)$ for a further $2 \mathrm{~min}$. This method

197 is the standard in eDNA extraction for endophyte research (e.g. Izuno et al. 2016, Haruna et

198 al. 2018), proving efficient in removing the maximum amount of epiphytic fungi and bacteria

199 from the leaf surface. Finally, leaves were dried with tissue paper, and stored in Ziploc bags

200 containing silica gel before transfer to the lab for DNA extraction.

201 DNA Isolation, PCR amplification and sequencing

202 Two $0.5 \mathrm{~cm}^{2}$ pieces of each leaf were placed into a 96 well plate, and a $3 \mathrm{~mm}$ aluminium bead

203 was added to each well. To facilitate the grinding step, the sealed plates were placed in a -80

$204{ }^{\circ} \mathrm{C}$ freezer for at least an hour before grinding using a Tissue Lyser (Qiagen, Germany) for 45

$205 \mathrm{sec}$ at $30 \mathrm{~Hz}$, followed by a centrifugation for 30 seconds at $6000 \mathrm{x} \mathrm{g}$. DNA was extracted

206 from samples using a NucleoSpin Plant II (96) kit (Macherey-Nagel, Düren, Germany)

207 according to the manufacturer protocol modifying only cell lysis time to $1 \mathrm{~h}$ and elution

208 volume to $100 \mu \mathrm{L}$.

209 PCRs were performed using two primers; ITS1 nuclear rDNA primers to target fungi

210 (Fwd: ITS5 GGAAGTAAAAGTCGTAACAAGG (Epp et al. 2012) and a modified version

211 of Rev: 5.8S_Fungi CAAGAGATCCGTTGTTGAAAGTK, Taberlet et al. 2018), and 16S

212 rDNA (V5-V6) primers to target bacteria (Bact01 primers - Fwd: 
213 GGATTAGATACCCTGGTAGT and Rev: CACGACACGAGCTGACG (Fliegerova et al.

214 2014)). To discriminate samples after sequencing, forward and reverse primers were

215 synthetized with a combination of two-different 8-nucleotide tags per sample, following a

216 double-indexing strategy (Binladen et al. 2007). As such, each PCR was amplified with a

217 unique combination of tagged primers. Each PCR reaction was performed in a total volume of

$21820 \mu \mathrm{l}$ and comprised $10 \mu \mathrm{l}$ of AmpliTaq GoldMaster Mix (Life Technologies, Carlsbad, CA,

219 USA), $5.84 \mu \mathrm{L}$ of nuclease-free water Ambion ${ }^{\mathrm{TM}}$ (Invitrogen, Waltham, Massachusetts,

220 USA), $0.25 \mu \mathrm{M}$ of each primer, $3.2 \mu \mathrm{g}$ of BSA (Roche Diagnostic, Basel, Switzerland), and 2

$221 \mu$ l of DNA template. Two PCRs per sample were performed under the following conditions:

222 Polymerase reactivation $10 \mathrm{~min}$ at $95^{\circ} \mathrm{C}$, followed by 35 (fungi) or 30 (bacteria) cycles of 30

$223 \mathrm{sec}$ at $95^{\circ} \mathrm{C}, 30 \mathrm{sec}$ at $55^{\circ} \mathrm{C}$ (fungi) or $57^{\circ} \mathrm{C}$ (bacteria) and $1 \mathrm{~min}$ at $72^{\circ} \mathrm{C}$; followed by a final

224 step of $7 \mathrm{~min}$ at $72^{\circ} \mathrm{C}$. Two wells in each plate were filled with water, to act as PCR negative

225 controls. Eight wells positioned randomly across each plate were left empty (with no PCR

226 products) to act as sequencing controls (non-used tag combinations).

227 Amplicons were pooled and libraries were prepared with the TruSeqNano PCR free

228 Illumina kit and were sequenced on Paired-end (2X250 bp) in the Illumina Miseq platform

229 (Illumina, San Diego, CA, USA) at the INRA Genotoul-GetPlaGe core facility (Toulouse,

230 France) using the Paired-end MiSeq Reagent Kit V3 (Illumina, San Diego, CA, USA),

231 following the manufacturer's instructions.

232 Sequence data curation

233 The generated reads were subject to a data-curation pipeline using scripts in $\mathrm{R}(\mathrm{R}$

234 Development Core Team, 2013), and the OBITools package (Boyer et al. 2016). Firstly,

235 'Illuminapairedend' was used for paired-end read assembly where an exact alignment

236 algorithm assigns a quality score for each nucleotide position, generating a score for each read

237 based on the number of mismatches, followed by 'ngsfilter' which removes primer and tag

238 sequences and assigns reads to samples. Here, reads with any primers with more than two

239 mismatches, and any tags with 1 or more mismatches are removed. 'obiuniq' then

240 dereplicates only those sequences which are strictly identical. 'obigreb' removes sequences of

241 low quality (containing Ns or with a pair alignment score below 50). Finally sequences with

242 only one read across the global dataset (singletons) were removed, since these represent

243 potential sequencing artefacts. 'sumaclust' was then used to generate Operational Taxonomic

244 Units (OTUs), where pairwise sequence dissimilarity using the raw number of mismatches

This article is protected by copyright. All rights reserved 
245 was calculated, and where those sequences $\geq 97 \%$ or more were assigned to the same OTU, a

246 threshold commonly applied to delimitate proxies for during molecular analysis of fungi in

247 particular (Nilsson et al., 2008; Coissac 2012). For each OTU, the sequence with the highest

248 number of reads in each cluster was assigned as the seed sequence, used for subsequent

249 taxonomic assignment.

250 Taxonomic assignment of OTUs was conducted using reference databases with the

251 'ecotag' function, which uses global alignment of sequences against full-length references.

252 For fungi the reference database was obtained by running an in-silico PCR with the ecoPCR

253 program (Ficetola et al., 2010) on Genbank (release 197; ftp://ftp.ncbi.nlm.nih.gov/genbank)

254 using the primer pairs employed here. Taxonomic assignment yielding the highest similarity

255 score was kept, with similarity scores for fungi ranging from 0.5 to 1 with sequences in ncbi.

256 For Bacteria, taxonomic assignment was conducted using the SILVA database (Quast et al.

257 2012), with similarity parameters kept at 0.97 , higher than fungi since bacterial sequence

258 databases are much more complete than for fungi (Truong et al., 2017). This approach

259 allowed for the elimination of a certain number of chimeras which are likely to have formed

260 during PCR. In particular, taxonomic identification facilitated later analysis, by distinguishing

261 between orders known to contain OTUs corresponding to endophytic fungi (Xylariales,

262 Capnodiales, Chaetothyriales, Phyllachorales, Diaporthales, Sordariales, Pleosporales,

263 Hypocreales) against orders which are known to contain generalist saprotrophs (Polyporales,

264 Eurotiales, Corticiales). Similarly, bacterial endophyte orders which have strict relationships

265 with plants (e.g. Rhizobiales, Acetobacterales, Sphingomonadales) could be compared with

266 orders known to contain more generalist bacteria (e.g. Solirubrobacterales,

267 Pseudonocardiales, Acidobacteriales).

268 Although initial processing of sequences using OBITools removes a large number of

269 erroneous sequences introduced to the dataset during sequencing, further processing based on

270 methodological controls can remove additional erroneous sequences and contaminants. OTUs

271 which could represent contaminants arising during extraction and during PCR (present within

272 the PCR negative controls) were removed from the dataset by comparing occurrence in

273 controls with that in samples. Potential contaminants were detected by calculating the mean

274 sum of reads for each OTU in PCR negative controls, and sequencing controls (blanks)

275 divided by the mean sum of reads across both these controls and samples. For each OTU, the

276 acceptable level of OTU abundance present in PCR negative controls was set to be 5\% or less 
277 than the total number of reads. Above this threshold, an OTU was more likely to be a

278 contaminant, and as such it was excluded. OTUs which only occur in negative controls were

279 also removed. Finally, all sequence data associated with blank controls was removed from the 280 datasets.

281 All OTUs which were identified as non-fungal following taxonomic assignment were 282 removed from the fungal database (S.I. 2). Bacterial sequences which were identified as 283 originating from ribosomes or chloroplasts were removed from the bacterial dataset, since 284 these likely represented plant host genetic material rather than that of bacterial endophytes 285 (S.I. 3). It is noted that future studies of bacterial endophytes should use primers more adapted 286 to reduce this effect (e.g. Beckers et al. 2016). PCR replicates were then pooled. We excluded

287 fungal samples with less than 100 reads from subsequent analysis. These low-quality samples 288 likely result from PCR amplification failure. This filter was chosen as a result of the majority 289 of samples averaging a much higher read count. This resulted in the removal of 14 samples, 290 with subsequent analysis for the fungal communities performed on a dataset of 351 leaves. In 291 comparison, bacterial read counts per sample were dominated by ribosome and chloroplast 292 sequences. Once these were removed, all samples containing a minimum of 1 read were kept 293 in the dataset, resulting in the removal of 8 leaves, with subsequent analysis for the bacterial 294 communities performed on a dataset of 357 leaves.

295 Following clustering of sequence reads with a 97\% similarity cut-off, 11,026 OTUs $296(67,338,264$ reads) were obtained for fungi, and 2,859 OTUs for bacteria (12,746,769 reads). 297 Removing OTUs occurring disproportionately in the negative controls and blank PCR wells 298 brought the number of OTUs down to 10,303 for fungi, and to 2,438 for bacteria. Removal of 299 non-fungal OTUs brought the total number of OTUs down to 10,160 (S.I. 2), and 1,953 OTUs 300 for bacteria (S.I 3). Subsequent analysis was performed on these reduced datasets.

301 Statistical analysis

302 In order to establish the relative effects of host tree niche creation and dispersal limitation,

303 leaf sample endophyte diversity was classified and community composition at multiple spatial 304 and phylogenetic scales was determined. All statistical analysis was performed using Rstudio 305 version 1.1.442 (R Development Core Team, 2013). 
To quantify endophyte diversity, rarefication curves for each PCR based on the

307 number of reads were compiled using the 'rarefy' function in the vegan package (Oksanen et

308 al. 2013). These curves demonstrated whether sequencing depth was sufficient in representing

309 the diversity of selected host tree endophyte diversity, defined by whether curves reached

310 asymptote. Species accumulation curves were also prepared using the 'specaccum' function,

311 which determine whether the number of leaves sampled for each species was sufficient in

312 capturing endophyte diversity. Subsequent analysis was conducted on a standardised dataset,

313 whereby the observed dataset was resampled so as to have the same relative abundance of

314 OTUs, but for read count per sample to be equal, using the first quantile of the dataset as the

315 new total read count per sample. Endophyte alpha diversity for each of the datasets (351

316 leaves for fungi, 357 for bacteria) was characterized by calculating a Shannon Index for each

317 sample, selected to neither over nor under represent rare OTUs. This was converted to

318 effective number of species, to reflect the number of equally-common species required to give

319 a particular value (Jost 2006).

320 The effect of host tree species in influencing endophyte alpha diversity was

321 determined using ANOVA, with Tukey Post Hoc tests revealing which species-by-species

322 comparisons were significant. To further understand within communities which OTU groups

323 differed significantly from one host tree species to the next, data were converted to presence

324 absence, and aggregated at the order level, chosen since taxonomic assignment for fungi in

325 particular was best at this level, and to allow for enough replication to statistically test for

326 patterns of variation, using ANOVA.

327 To determine host species effects on community composition and beta diversity,

328 variability in endophyte community composition was examined across all 351 leaf samples.

329 Bray-Curtis similarity measures were used to construct a community distance matrix based on

330 the observed endophyte communities within each sample. The variance in distance between

331 groups of interest was compared with a nonparametric null distribution based on 999

332 permutations of the observed data. The effect of host tree species on endophyte community

333 composition was tested on the complete dataset ( $n=351$ for fungi, 357 for bacteria). The

334 effect of individual host tree, and branch within each tree on endophyte community

335 composition was tested on a reduced similarity matrix composed of samples collected from

336 only 3 branches per tree ( $n=327$ for fungi, 331 for bacteria). These were evaluated with

337 permutational multivariate analysis of variance (PERMANOVA; Anderson 2013), using the

338 'adonis' function in the vegan package (Oksanen et al. 2013). To visualise the variation 
339 explained by host tree individual, host tree species, and branch identity, partitioning of

340 variance was conducted using the 'varpart' function in vegan.

341 To explore whether observed variance was common across all fungal endophytes, or 342 whether it differed across OTUs within different orders PERMANOVA were performed on

343 two subsets of the data; one containing only OTUs identified as belonging to the order 344 Xylariales (well documented endophytes, see Rodriguez et al., 2009), the other containing

345 OTUs identified to order Polyporales (order known to contain pathogens). Similarly, for

346 bacteria, analysis was rerun on a subset of the data, comprising those OTUs identified to the

347 order Rhizobiales (an order known to contain plant associated bacteria) and another data set 348 comprised of OTUs identified to the order Solirubrobacterales (an order known to contain 349 generalist bacteria).

350 To determine the influence of phylogenetic and geographic distance of the host tree 351 species on the endophytic communities, OTU reads for each sample from the same tree were

352 summed, reducing the full datasets for both fungi and bacteria down to 38 samples. DNA read

353 counts were normalised across all trees so as to have a balanced dataset. Bray-Curtis

354 similarity of each tree endophyte community was determined and compiled in a community

355 similarity matrix. A phylogenetic tree representing all of the species sampled here was

356 prepared using the ape package in R (Fig. 3; Paradis et al. 2004), by pruning a species-level

357 phylogenetic hypothesis published elsewhere (Baraloto et al. 2012). Terminal nodes

358 representing members of the same species, i.e. where more than one tree was sampled, were

359 included as polytomies with an intraspecific edge distance of 0.5 between all, so as to be very

360 low in comparison to the interspecific edge distances elsewhere in the phylogeny. The

361 resulting edge distances were then used to prepare a phylogenetic distance matrix.

362 A second distance matrix was prepared comparing the geographic distance between

363 each of the 38 trees across the 4-ha sampling plot in metres. Both matrices were directly

364 compared with the endophyte community similarity matrices by performing partial Mantel

365 tests based on Pearson's product-moment correlation, using the function mantel.partial in the

366 ecodist package (Goslee \& Urban 2007). In each case, the effect of one distance metric was

367 controlled for when determining the effect of the other.

In order to determine whether the presence of OTUs within the leaves of a host tree

369 represented true host specificity, and to avoid issues associated with low sample size (e.g. 
370 Cordier et al. 2012, Novotny et al. 2002), a metric was calculated to determine the probability

371 that OTU presence within a single host species occurred by chance. Using the bipartite

372 package (Dormann et al. 2008), the degree of each OTU (i.e. the number of leaf samples

373 within which they occur) was calculated. Host tree name was replaced by a numerical value

374 (1-22 for each sampled host), before a random sample of host tree for each of these degrees

375 was determined. The standard deviation of the randomly generated host tree numbers was

376 then calculated. Each time this equalled zero (i.e. the degrees were sampled as the same

377 species), a count of 1 was assigned. This process was reiterated 1000 times, with the count

378 tally of iterations divided by 1000 . The calculated metric thus ranged from $0-1$, with 0

379 representing no chance that an OTU occurred in sampled host by chance, and 1 representing

380 certainty that OTUs occurred in sampled host by chance. For OTUs with a degree of 1, such

381 a calculation was not possible. To determine the importance of rare OTUs in generating

382 patterns detected during analysis, the percentage of all OTUs only occurring in each host tree

383 was calculated.

384 Results

385 Endophyte $\alpha$-diversity

386 Average OTU richness per leaf was calculated to be 168 for fungi, ranging from 15 to 1018 ,

387 and 46.3 for bacteria, ranging from 1 to 504. Fungal OTU identification rate was 45\%, 36\%,

388 and $19 \%$ at the order, family, and genus level, respectively. Bacterial OTU identification was

$38998 \%, 88 \%$, and $59 \%$ at the order, family, and genus level, respectively. OTU accumulation

390 curves indicated that sampling was representative of the total endophyte community for fungi,

391 but to a lesser extent for bacteria (S.I.4). Sequencing depth varied across samples (see S.I. 5),

392 justifying standardisation approaches when calculating alpha diversity metrics.

393 Per-leaf endophyte diversity was dependent on the host tree with above average

394 numbers of OTUs only occurring in specific host trees (Fig. 2). Significant differences in host

395 tree Shannon diversity indices were detected for both fungi (ANOVA F $21,335=4.42, p=$

$396<0.001$ ), and to a lesser extent for bacteria (ANOVA F $21,336=1.82 \mathrm{p}=0.016$. Tukey HSD

397 revealed that leaves collected from Carapa surinamensis, Dussia discolor, Inga huberi and

398 Virola kwatae had significantly higher diversity indices than those collected from other trees

399 for fungi, whilst for bacteria, only Virola kwatae was found to be significantly higher in 
400 diversity than Eperua rubiginosa, with all other host species not differing significantly in their 401 bacterial diversity (Fig. 2).

402 The relative occurrence of certain fungal orders (using OTU occurrence aggregated 403 for each order) differed significantly from one host tree to the next (Fig. 3; S.I. 6).

404 Host-tree effects on $\beta$-diversity

405 When examining the effects of host tree identity on leaf endophyte community composition, 406 PERMANOVA revealed the significant contribution of both species (Fungi: $\mathrm{F}=2.65, \mathrm{p}=$ 407 0.001; Bacteria: $\mathrm{F}=1.97, \mathrm{p}=0.001$ ) and nested individual trees (Fungi: $\mathrm{F}=1.58, \mathrm{p}=0.001$; 408 Bacteria: $\mathrm{F}=1.3, \mathrm{p}=0.001$ ) in contributing to variation (Table 1). In addition to the importance of individual host tree (Fungi: $F=2.25, p=0.001$; 410 Bacteria: $\mathrm{F}=1.70, \mathrm{p}=0.001$ ), PERMANOVA also demonstrated a significant effect of 411 nested branch (Fungi: $F=1.08, \mathrm{p}=0.001$; Bacteria: $\mathrm{F}=1.12, \mathrm{p}=0.001$ ) in determining 412 endophyte community composition (Table 2). Although host tree is a significant contributor, 413 it only explains a part of the variation amongst communities, as revealed by the associated R2 414 values (Table 1). Partitioning of variance demonstrated the cumulative effects of species, host 415 individual and branch only accounted for $12 \%$ of variation for fungi, and $10 \%$ of variation for 416 bacteria (Fig. 4)

417 Endophyte order-specific effects on $\beta$-diversity

418 When determining the effects of host tree species on specific orders of fungi, PERMANOVA 419 demonstrated a significant effect for Xylariales $(\mathrm{F}=1.78, \mathrm{p}=0.001$; Table 1$)$ but not for 420 Polyporales $(\mathrm{F}=1.01, \mathrm{p}=0.434$; Table 1$)$. When determining the effects of host tree species 421 on specific orders of bacteria, PERMANOVA demonstrated a significant effect for both 422 Rhizobiales $(F=1.92, \mathrm{p}=0.001$; Table 1$)$ and Solirubrobacterales $(\mathrm{F}=1.5, \mathrm{p}=0.007$; Table 423 1).

424 Phylogenetic and geographic effects on $\beta$-diversity

425 Mantel tests revealed a significant correlation between endophyte community composition 426 and phylogenetic distance of the host tree species for both fungi $(r=0.1251, p=0.044)$, and 427 bacteria $(r=0.227, p=0.009)$. Both groups displayed no significant correlation between tree 
428 endophyte community composition and geographic distance (Fungi: $r=0.091, p=0.064$;

429 Bacteria: $\mathrm{r}=-0.062, \mathrm{p}=0.783$ ).

\section{$430 \quad$ OTU host specificity}

431 Out of the 10158 detected fungal OTUs, 5542 occurred in only one host tree. Of these, 4982

432 only occurred in one leaf and as such host specificity could not be determined. For the

433 remaining 560 OTUs, when accounting for the probability that their occurrence within a host

434 was determined by chance with a cut off of 0.05 , only 81 OTUs demonstrated true host

435 specificity. This usually corresponded to OTUs occurring in 3 or more leaves of host trees of

436 the same species, encompassing a broad taxonomy of fungal species (S. I. 7).

437

438 Out of the 1954 detected bacterial OTUs, 901 occurred in only one host tree. Of these, 737

439 only occurred in one leaf and as such host specificity could not be determined. For the

440 remaining 164 OTUs, when accounting for the probability that their occurrence within a host

441 was determined by chance with a cut off of 0.05 , only 14 OTUs demonstrated true host

442 specificity. This usually corresponded to OTUs occurring in 3 or more leaves of host trees of

443 the same species, encompassing a broad taxonomy of bacterial species (S. I. 7).

445 Conversely, no single OTU occurred across all sampled leaves, and only 31 fungal OTUs and 4463 bacterial OTUs occurred in more than half of the total leaf number.

447

\section{Discussion}

449 Tropical foliar endophytes represent an ideal model system to explore the factors shaping

450 ecological communities. The high levels of both $\alpha$ - and $\beta$-diversity found in this study reveal 451 the relative importance of host tree contributions to endophyte niche and dispersal limitation 452 across spatial scales. In spite of acknowledged biases introduced by PCR methods and 453 clustering thresholds (Dickie 2010, Lindner \& Banik 2011), and a potentially low level of

454 bacteria detection as a result of a primer which also amplified chloroplast and mitochondrial 455 sequences, eDNA methods used here reveal levels of endophyte diversity an order of 456 magnitude higher than culture-based methods, with OTU taxonomic identification and 457 relative abundance consistent with previous studies of tropical canopy microbial communities 458 (e.g. Izuno et al. 2016, Haruna et al. 2018). 
Differences in endophyte diversity and community composition were detected at the

460 host tree level for fungi. Given this trend was consistent with tree phylogenetic relatedness, it

461 is likely that endophyte colonization success is dependent on shifts in leaf traits across host

462 tree species, such as cuticle thickness or tannin levels (Arnold and Herre 2003). Carapa

463 surinamensis, Dussia discolor, Inga huberi and Virola kwatae trees displayed higher levels of

464 fungal endophyte diversity than other species present under similar conditions, and given the

465 known role of certain tropical endophytes as symbionts (e.g. Arnold et al. 2003; Bae et al.

466 2009; Van Bael et al. 2009; Christian et al. 2019), such variation in the endophytes present

467 could have functional consequences on host plant health for canopy leaves.

468 When observing the diversity of endophytes associated with host tree species at the

469 order level, clear differences in the relative abundance of certain groups are apparent. Certain

470 orders of endophytes differed significantly across different host species (e.g. Xylariales, for

471 fungi; Rhizobiales for bacteria), whilst orders characterized by OTUs known to occur across a

472 wide range of habitat types (e.g. Polyporales for fungi; Solirubrobacterales for bacteria) did

473 not differ significantly in their occurrence among host tree species. Such a finding mirrors

474 that of Peay et al. (2013), which showed that soil fungal communities with lineages

475 containing pathogens and mycorrhizal fungi correlated with tree species richness of tropical

476 forest plots, whilst saprotrophs did not. Nevertheless, at the 4-ha plot scale, although

477 significant, host tree only explained a small proportion of the variation in endophyte

478 community composition, suggesting the contribution of other factors.

479 Limitations in dispersal ability are known to have consequences in structuring spore

480 dependent ecological communities (Peay et al. 2012). Here, distance between leaves was

481 found to result in significant differences in endophyte community assembly, although this was

482 scale dependent. When aggregating samples at the host tree individual level, no significant

483 correlation was found between tree endophyte community composition and their proximity to

484 one another within the 4-ha plot. At the scale of a single tree crown however, leaves

485 positioned on the same branch contained endophyte communities more similar to one another

486 than those found on an adjacent branch. This result could reflect differences in the age of

487 leaves, given that leaves occurring on the same branch will be more similar in age, with

488 consequences for leaf chemistry and community establishment. The result could also suggest

489 an effect of dispersal limitation in determining endophyte community composition within a

490 tropical forest upper canopy, albeit only detectable at scales of less than $10 \mathrm{~m}$. Beyond this, in

491 absence of the physical limitations to dispersal such as in the understory (e.g. Arnold et al. 
492 2003, Izuno et al. 2016), of a strong environmental gradients (e.g. Suryanarayanan et al. 2002,

493 Zimmerman \& Vitousek 2012), and because of high rates of spore dispersal (e.g. Peay et al.

494 2012) tropical forest canopies are likely to display communities characterised by high turn-

495 over. This contrasts with the findings of low species turnover at both local (10-100m) and

496 regional scales $(100 \mathrm{~km})$ in a tropical forest understory for fungal endophytes (Vincent et al.

497 2016). Here, authors used culturing to detect fungal OTUs and detected high numbers of

498 generalist OTUs across their sampled host trees and sites. In comparison, our use of

499 metabarcoding fails to detect high numbers of generalists, and instead reveals the importance

500 of large numbers of rare OTUs.

501 Partitioning of the variance in endophyte community composition revealed that at the

502 canopy scale, only a small part of total variation in endophyte assemblage could be explained

503 by the summed effects of branch, host tree, and host species (12\% for fungi, $10 \%$ for

504 bacteria). Thus, although significant, each of these factors are limited in explaining the high

505 levels of variation in the system. Instead, leaf endophyte assemblages are largely

506 characterized by rare OTUs which occur sporadically within samples, the sum of which

507 represents a large part of the total diversity within the canopy. Indeed, when accounting for

508 the probability of chance in determining the presence of an OTU within a host tree, for both

509 fungi and bacteria only $0.7 \%$ of detected OTUs could truly be considered host specific.

510 Conversely, no OTUs were so generalist that they occurred across all samples, and only a

511 small proportion occurred in more than half of all sampled leaves. In spite of the relatively

512 small sample size here, this finding counters the idea of a 'core microbiome' for endophytes

513 (e.g. Kembel et al. 2014), at least in the context of this study. Thus, the detected variation

514 across host species is likely to be driven by the differences in the relative abundance of a

515 small number of generalist OTUs and the numerous rare OTUs.

516 Due to their presence in well-defined sampling units, foliar endophytes represent an

517 ideal example of community assembly within which to test assembly rules for betadiversity in

518 tropical habitats. Communities associated with leaves collected in the upper canopy are

519 known to be distinct from those found at lower levels (Izuno et al. 2016), but the findings

520 presented here demonstrate the scale dependant factors contributing to their composition.

521 When carefully controlling for community scale, and sampling intensity, community

522 assembly becomes clearly non-neutral in leaf-associated fungi and bacteria, but not in

523 opportunistic species which may tend to be rare and found across a range of samples

524 (Magurran \& Henderson 2003). Given the emerging knowledge surrounding their role as

This article is protected by copyright. All rights reserved 
symbionts, the community structure of endophytes within the canopy layer is likely to play an

526 important role in the non-negligible exchanges of energy and matter which define this habitat.

527 Acknowledgements - The Nouragues Ecological Research Station is supported by CNRS,

528 and is part of the Nouragues Natural Reserve. Fieldwork was supported by Patrick Châtelet

529 and Marie-Loréa Tapie-Petit. Valuable contributions to the manuscript were provided by

530 Lucie Zinger and other participants of the 2018 Metabarcoding School. We are grateful to the

531 Genotoul bioinformatics platform (Toulouse, Midi-Pyrénées) for providing computing

532 resources. This work was supported by a joint Agence Nationale de la Recherche-Swiss

533 National Science Foundation (ANR-SNF) grant (SECIL, ref ANR-15-CE21-0016 and SNF

$534 N^{\circ} 310030 \mathrm{E}-164289$ ) and from an "Investissement d'Avenir" grants also managed by Agence

535 Nationale de la Recherche (CEBA, ref. ANR-10-LABX-25-01; ANAEE-France: ANR-11-

536 INBS-0001, TULIP: ANR-10-LABX-0041).

537 Author contributions - All authors contributed to manuscript preparation; US, LP, PG and

538 JC conducted field work collection of samples; MR, US, AI and SM performed lab-based data

539 preparation; JD, MR and JC performed data analysis.

540 Data Archive - Data available from the Dryad Digital Repository:

541 https://doi.org/10.5061/dryad.XXXXXX

\section{References}

543 Anderson, M. J. and Walsh, D. C. 2013. PERMANOVA, ANOSIM, and the Mantel test in the

544 face of heterogeneous dispersions: what null hypothesis are you testing? - Ecological

545 Monographs 83: 557-574.

546 Arnold, A. E. and Engelbrecht, B. M. 2007. Fungal endophytes nearly double minimum leaf

547 conductance in seedlings of a neotropical tree species. - Journal of Tropical Ecology 23: 369-

548372.

549 Arnold, A. E. and Herre, E. A. 2003. Canopy cover and leaf age affect colonization by

550 tropical fungal endophytes: ecological pattern and process in Theobroma cacao

551 (Malvaceae). - Mycologia 95: 388-398.

552 Arnold, A. E. and Lutzoni, F. 2007. Diversity and host range of foliar fungal endophytes: are

553 tropical leaves biodiversity hotspots? - Ecology 88: 541-549. 
554 Arnold, A. E. et al. 2000. Are tropical fungal endophytes hyperdiverse? - Ecology Letters 3: $555 \quad 267-274$.

556 Arnold, A. E. et al. 2003. Fungal endophytes limit pathogen damage in a tropical tree. 557 PNAS 100: 15649-15654.

558 Bae, H. et al. 2009. The beneficial endophyte Trichoderma hamatum isolate DIS 219b

559 promotes growth and delays the onset of the drought response in Theobroma cacao. - J. Exp.

560 Bot. 60: 3279-3295.

561 Baraloto, C. et al. 2012. Using functional traits and phylogenetic trees to examine the

562 assembly of tropical tree communities. - J. Ecology 100: 690-701.

563 Binladen J, et al. 2007. The use of coded PCR primers enables high-throughput sequencing of 564 multiple homolog amplification products by 454 parallel sequencing. PLoS ONE, 2: e197.

565 Boyer, F. et al. 2016. obitools: a unix-inspired software package for DNA metabarcoding. -

566 Mol Ecol Resour. 16: 176-182.

567 Carrell, A. and Carper, D. 2016. Subalpine conifers in different geo- graphical locations host 568 highly similar foliar bacterial endophyte communities. - FEMS Microbiol. Ecol. 92:fiw124

569 Carroll, G. 1988. Fungal endophytes in stems and leaves: from latent pathogen to mutualistic 570 symbiont. - Ecology 69: 2-9.

571 Chase, J. M. et al. 2005. Competing theories for competitive metacommunities. - In:

572 Holyoak, M., Leibold, M. A. and Holt, R. D. (eds), Metacommunities: spatial dynamics and 573 ecological communities. University of Chicago Press, pp. 335-354.

574 Christian, N. et al. 2019. Foliar endophytic fungi alter patterns of nitrogen uptake and

575 distribution in Theobroma cacao. - New Phytol. https://doi.org/10.1111/nph.15693

576 Coissac, E. et al. 2012. Bioinformatic challenges for DNA metabarcoding of plants and 577 animals. - Mol Ecol. 21: 1834-1847.

578 Condit, R. et al. 2002. Beta-diversity in tropical forest trees. - Science. 295: 666-669.

579 Cordier, T. et al. 2012. The composition of phyllosphere fungal assemblages of European

580 beech (Fagus sylvatica) varies significantly along an elevation gradient. - New Phytol. 196: $581-510-519$.

582 Courtois, E. A. et al. 2016. Evolutionary patterns of volatile terpene emissions across 202 583 tropical tree species. - Ecol. Evol 6: 2854-2864. 
584 Crowther, T. W. et al. 2018. The use of artificial media in fungal ecology. - Fung. Ecol. 32:

$585 \quad 87-91$.

586 Del Olmo-Ruiz, M. and Arnold, A. E. 2017. Community structure of fern-affiliated

587 endophytes in three neotropical forests. - J Trop Ecol. 33: 60-73.

588 Dickie, I. A. 2010. Insidious effects of sequencing errors on perceived diversity in molecular 589 surveys. - New Phytol. 188: 916-918.

590 Dormann, C. F. et al. 2008. Introducing the bipartite package: analysing ecological

591 networks. - Interaction, 8: 8-11.

592 Ellwood, M. D. F. et al. 2009. Stochastic and deterministic processes jointly structure tropical 593 arthropod communities. - Ecol Lett. 12: 277-84.

594 Epp, L. S. et al. 2012. New environmental metabarcodes for analysing soil DNA: potential for 595 studying past and present ecosystems. - Mol. Ecol. 21: 1821-1833.

596 Ficetola, G. F. et al. 2010. An in silico approach for the evaluation of DNA barcodes. - BMC

597 genomics. 11: 434.

598 Fine, P. V. et al. 2004. Herbivores promote habitat specialization by trees in Amazonian 599 forests. - Science. 305: 663-665.

600 Fliegerova, K. et al. 2014. Effect of DNA extraction and sample preservation method on 601 rumen bacterial population. - Anaerobe, 29: 80-84.

602 Gilbert, G. S. and Reynolds, D. R. 2005. Nocturnal fungi: Airborne spores in the canopy and 603 understory of a tropical rain forest. - Biotropica. 37: 462-464.

604 Goslee, S. C. and Urban, D. L. 2007. The ecodist package for dissimilarity-based analysis of 605 ecological data. - J. Stat. Softw. 22: 1-19.

606 Griffin, E. A. and Carson, W. P. 2015. The ecology and natural history of foliar bacteria with 607 a focus on tropical forests and agroecosystems. - Bot Rev. 81: 105-149.

608 Griffin, E. A. \& Carson, W. P. 2018. Tree Endophytes: Cryptic Drivers of Tropical Forest 609 Diversity. - In: Pirttilä, A. M. and Frank, C. (eds), Endophytes of Forest Trees: Biology and 610 Applications. Springer, pp. 63-103.

611 Haruna, E. et al. 2018. Extensive overlap of tropical rainforest bacterial endophytes between 612 soil, plant parts, and plant species. - Microb ecol. 75: 88-103. 
613 Herre, E.A. et al. 2007. Ecological implications of anti-pathogen effects of tropical fungal

614 endophytes and mycorrhizae. - Ecology. 3: 550-558.

615 Hubbell, S.P. 2001. The unified neutral theory of species abundance and diversity. -

616 Princeton Univ. Press.

617 Hyde, K. and Soytong, K. 2008. The fungal endophyte dilemma. - Fungal Divers. 33: e173.

618 Izuno, A. et al. 2016. Vertical Structure of Phyllosphere Fungal Communities in a Tropical

619 Forest in Thailand Uncovered by High-Throughput Sequencing. - PloS one. 11: e0166669.

620 Jost, L. 2006. Entropy and diversity. - Oikos. 113: 363-375.

621 Kembel, S. W. et al. 2014. Relationships between phyllosphere bacterial communities and

622 plant functional traits in a neotropical forest. PNAS. 111: 13715-13720.

623 Leibold, M. A. 1995. The niche concept revisited: mechanistic models and community

624 context. - Ecology. 76: 1371-1382.

625 Lindner, D. L. and Banik, M. T. 2011. Intragenomic variation in the ITS rDNA region

626 obscures phylogenetic relationships and inflates estimates of operational taxonomic units in

627 genus Laetiporus. - Mycologia. 103: 731-740.

628 Lodge, D. J. 1997. Factors related to diversity of decomposer fungi in tropical forests. -

629 Biodivers. Conserv. 6: 681-688.

630 Magurran, A. E. and Henderson, P. A. 2003. Explaining the excess of rare species in natural 631 species abundance distributions. - Nature. 422: 714-716.

632 Maynard, D. S. et al. 2017. Fungal interactions reduce carbon use efficiency. - Ecol Lett. 20: 633 1034-1042.

634 Mejía, L.C. et al. 2014. Pervasive effects of a dominant foliar endophytic fungus on host 635 genetic and phenotypic expression in a tropical tree. - Front. Microbiol. 5: 479.

636 Miyambo T et al. 2016. Plants of the fynbos biome harbour host species-specific bacterial 637 communities. - FEMS Microbiol. Lett. 363:fnw122.

638 Nilsson, R.H., et al. 2008. Intraspecific ITS variability in the kingdom Fungi as expressed in 639 the international sequence databases and its implications for molecular species identification. 640 - Evolutionary bioinformatics. 4: EBO-S653.

641 Novotny, V. et al. 2002. Low host specificity of herbivorous insects in a tropical forest. 642 Nature 416: 841-844. 
643 Oksanen, J. et al. 2013. Package ‘vegan', Community ecology package, version, vol. 2, no. 9.

644 Paradis, E. et al. 2004. APE: analyses of phylogenetics and evolution in R language. 645 Bioinformatics. 20: 289-290.

646 Peay, K. G. et al. 2013. Strong coupling of plant and fungal community structure across 647 western Amazonian rainforests. - ISME. 7: no. 9, pp. 1852.

648 Peay, K. G. et al. 2012. Measuring ectomycorrhizal fungal dispersal: macroecological patterns 649 driven by microscopic propagules. - Mol Ecol. 21: 4122-4136.

650 Porras-Alfaro, A. and Bayman, P. 2011. Hidden Fungi, Emergent Properties: Endophytes and 651 Microbiomes. - Annu. Rev. Phytopathol. 49:291-315.

652 Quast, C. et al. 2012. The SILVA ribosomal RNA gene database project: improved data 653 processing and web-based tools. - Nucleic Acids Res. 41: 590-596.

654 R Core Team. 2013. R: A language and environment for statistical computing, Vienna, 655 Austria.

656 Réjou-Méchain, M. et al. 2015. Using repeated small-footprint LiDAR acquisitions to infer 657 spatial and temporal variations of a high-biomass Neotropical forest. - Remote Sens Environ. 658 169: 93-101.

659 Ricklefs, R. E. 2004. A comprehensive framework for global patterns in biodiversity. - Ecol 660 Lett. 7: 1-15.

661 Rodriguez, R.J. et al. 2009. Fungal endophytes: diversity and functional roles. - New phytol. $662 \quad 182: 314-330$.

663 Sabatier, D. 1990. Variations du peuplement forestier à l'échelle stationnelle: le cas de la 664 station des Nouragues en Guyane Francaise. - Actes de l'Atelier MAB-UNESCO sur 665 l'Aménagement et la Conservation de l'Ecosystème Forestier Humide.

666 Suryanarayanan, T. et al. 2002. Occurrence and distribution of fungal endophytes in tropical 667 forests across a rainfall gradient. - Can J Bot. 80: 818-826.

668 Suryanarayanan, T. et al. 2003. Endophytic fungal communities in leaves of tropical forest 669 trees: diversity and distribution patterns. - Curr. Sci. 85: 489-493.

670 Taberlet, P. et al. 2018. Environmental DNA: For Biodiversity Research and Monitoring. 671 Oxford University Press. 
672 Truong, C. et al. 2017. How to know the fungi: combining field inventories and 673 DNA-barcoding to document fungal diversity. - New Phytol. 214: 913-919.

674 U'Ren, J. M. et al. 2012. Host and geographic structure of endophytic and endolichenic fungi 675 at a continental scale. - Am. J. Bot. 99: 898-914.

676 Van Bael, S. et al. 2017. Foliar endophyte communities and leaf traits in tropical trees. In:

677 The fungal community: its organization and role in the ecosystem. CRC Press, Boca Raton, 678 pp.79-94.

679 van der Meer, P. J. and Bongers, F. 1996. Patterns of tree-fall and branch-fall in a tropical rain 680 forest in French Guiana. - J. Ecology 84:19-29.

681 van der Meer, J. H. 1972. Niche theory. - Annu. Rev. Ecol. Evol. Syst. 3:107-132.

682 Vincent, J. B. et al. 2016. Host associations and beta diversity of fungal endophyte 683 communities in New Guinea rainforest trees. - Mol. Ecol. 25: 825-841.

684 Zimmerman, N. B. and Vitousek, P. M. 2012. Fungal endophyte communities reflect 685 environmental structuring across a Hawaiian landscape. - PNAS. 109: 13022-13027.

686 Zinger, L. et al. 2016. Extracellular DNA extraction is a fast, cheap and reliable alternative for 687 multi-taxa surveys based on soil DNA. - Soil Biol. Biochem. 96: 16-1 
Table 1: PERMANOVA analysis to determine the effect of host tree species and nested host tree individual in determining fungal and bacterial endophyte community composition. Analysis was performed on community dissimilarity matrixes constructed from six separate datasets; one containing all fungal OTUs detected in the analysis of 351 leaves; one containing only OTUs identified as Xylariales; one containing only OTUs identified as Polyporales; one containing all bacterial OTUs detected in the analysis of 358 leaves; one containing only OTUs identified as Rhizobiales and one containing only OTUs identified as Solirubrobacterales.

\begin{tabular}{|c|c|c|c|c|c|c|c|c|c|c|c|c|c|c|c|c|c|c|c|c|c|c|c|c|c|c|}
\hline & \multirow{6}{*}{$\begin{array}{c}\overline{60} \\
\Xi \\
\Xi\end{array}$} & \multicolumn{4}{|c|}{351 leaf data } & \multicolumn{4}{|c|}{ Xylariales data } & \multicolumn{4}{|c|}{ Polyporales data } & \multirow{6}{*}{ 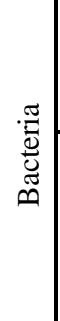 } & \multicolumn{4}{|c|}{358 leaf data } & \multicolumn{4}{|c|}{ Rhizobiales data } & \multicolumn{4}{|c|}{ Solirubrobacterales } \\
\hline & & $\mathrm{df}$ & $\mathrm{F}$ & $\mathrm{R} 2$ & $\mathrm{p}$ & df & $\mathrm{F}$ & $\mathrm{R} 2$ & $\mathrm{p}$ & $\mathrm{df}$ & $\mathrm{F}$ & $\mathrm{R} 2$ & $\mathrm{p}$ & & df & $\mathrm{F}$ & $\mathrm{R} 2$ & $\mathrm{p}$ & $\mathrm{df}$ & $\mathrm{F}$ & $\mathrm{R} 2$ & $\mathrm{p}$ & df & $\mathrm{F}$ & $\mathrm{R} 2$ & $\mathrm{p}$ \\
\hline $\mathrm{Sp}$ & & 21 & 2.65 & 0.14 & 0.001 & 21 & 1.78 & 0.11 & 0.001 & 21 & 1.01 & 0.12 & 0.434 & & 21 & 1.97 & 0.11 & 0.001 & 21 & 1.92 & 0.18 & 0.001 & 15 & 1.5 & 0.35 & 0.007 \\
\hline Sp: Tree & & 16 & 1.58 & 0.06 & 0.001 & 16 & 1.32 & 0.06 & 0.001 & 16 & 0.91 & 0.09 & 0.855 & & 16 & 1.3 & 0.05 & 0.001 & 15 & 1.28 & 0.09 & 0.001 & 9 & 1.09 & 0.15 & 0.336 \\
\hline Residuals & & 313 & & 0.79 & & 287 & & 0.83 & & 134 & & 0.79 & & & 320 & & 0.83 & & 166 & & 0.73 & & 31 & & 0.5 & \\
\hline Total & & 350 & & 1 & & 324 & & 1 & & 171 & & 1 & & & 357 & & 1 & & 202 & & 1 & & 55 & & 1 & \\
\hline
\end{tabular}


Table 2: PERMANOVA analysis to determine the effect of host tree individual and nested branch in determining endophyte community composition. Analysis was performed on community dissimilarity matrixes constructed from a dataset comprised of 327 leaves for fungi, 331 for bacteria.

\begin{tabular}{l|rrrr|rrrr}
\multicolumn{5}{c|}{ Fungi } & \multicolumn{4}{c}{ Bacteria } \\
\hline & df & F & R2 & p & df & F & R2 & p \\
\hline Tree & 37 & 2.25 & 0.22 & .001 & 37 & 1.70 & 0.17 & .001 \\
Tree: Branch & 75 & 1.08 & 0.21 & .001 & 75 & 1.12 & 0.23 & .001 \\
Residuals & 214 & & 0.57 & & 218 & & 0.60 & \\
Total & 326 & & 1 & & 330 & & 1 & \\
\hline
\end{tabular}


Figure 1: The Canopy Operational Permanent Access (COPAS) system, allowing access for unrestricted access to the upper canopy. This was used to sample leaves from which endophyte communities were extracted.

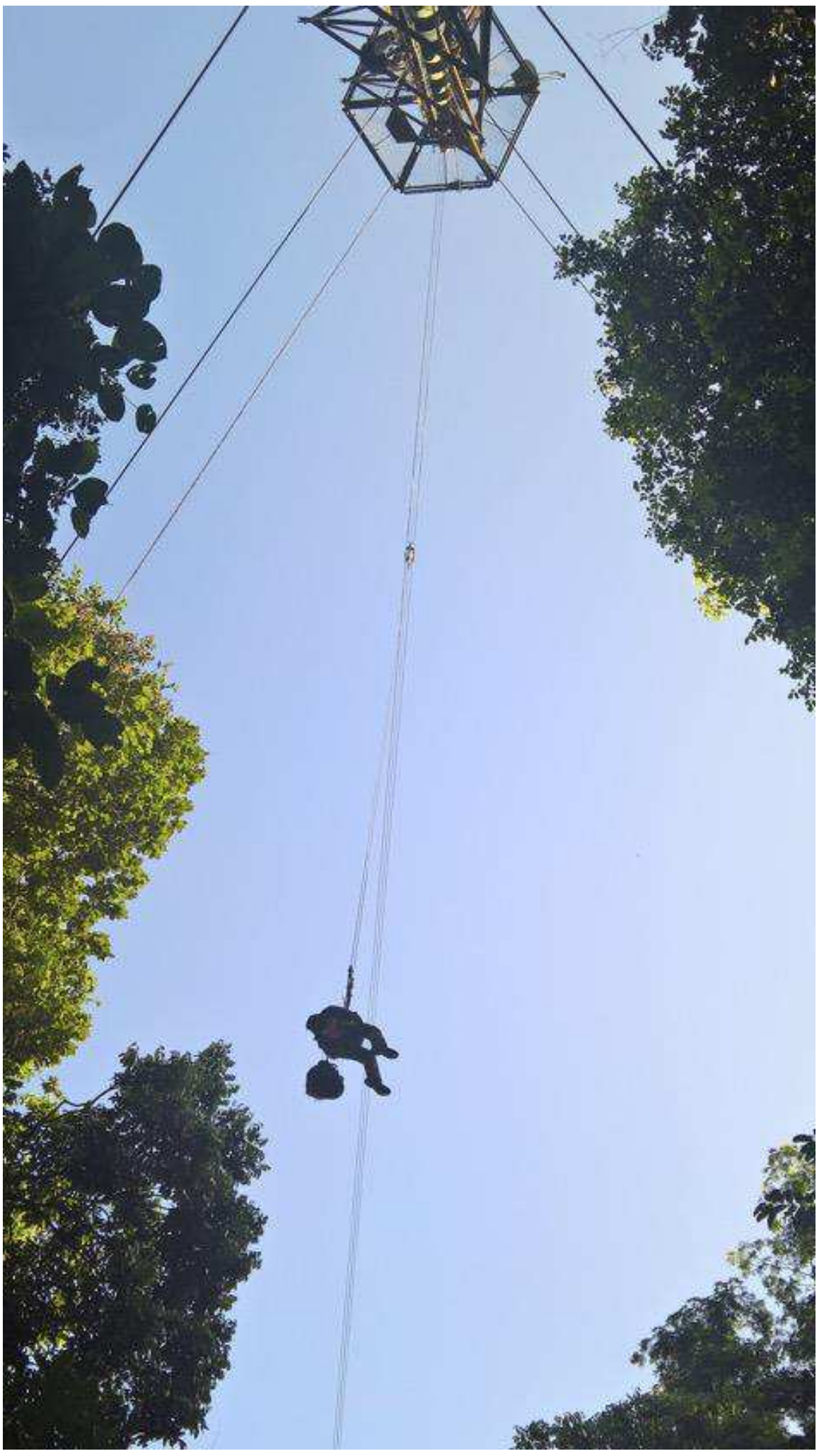

This article is protected by copyright. All rights reserved 
Figure 2: The effective Shannon diversity index of each sampled tree species, based on the of OTUs within each leaf sample. The fungal dataset is based on 351 leaves and the bacterial dataset is based on 357 leaves. Trees are arranged by phylogenetic relatedness.

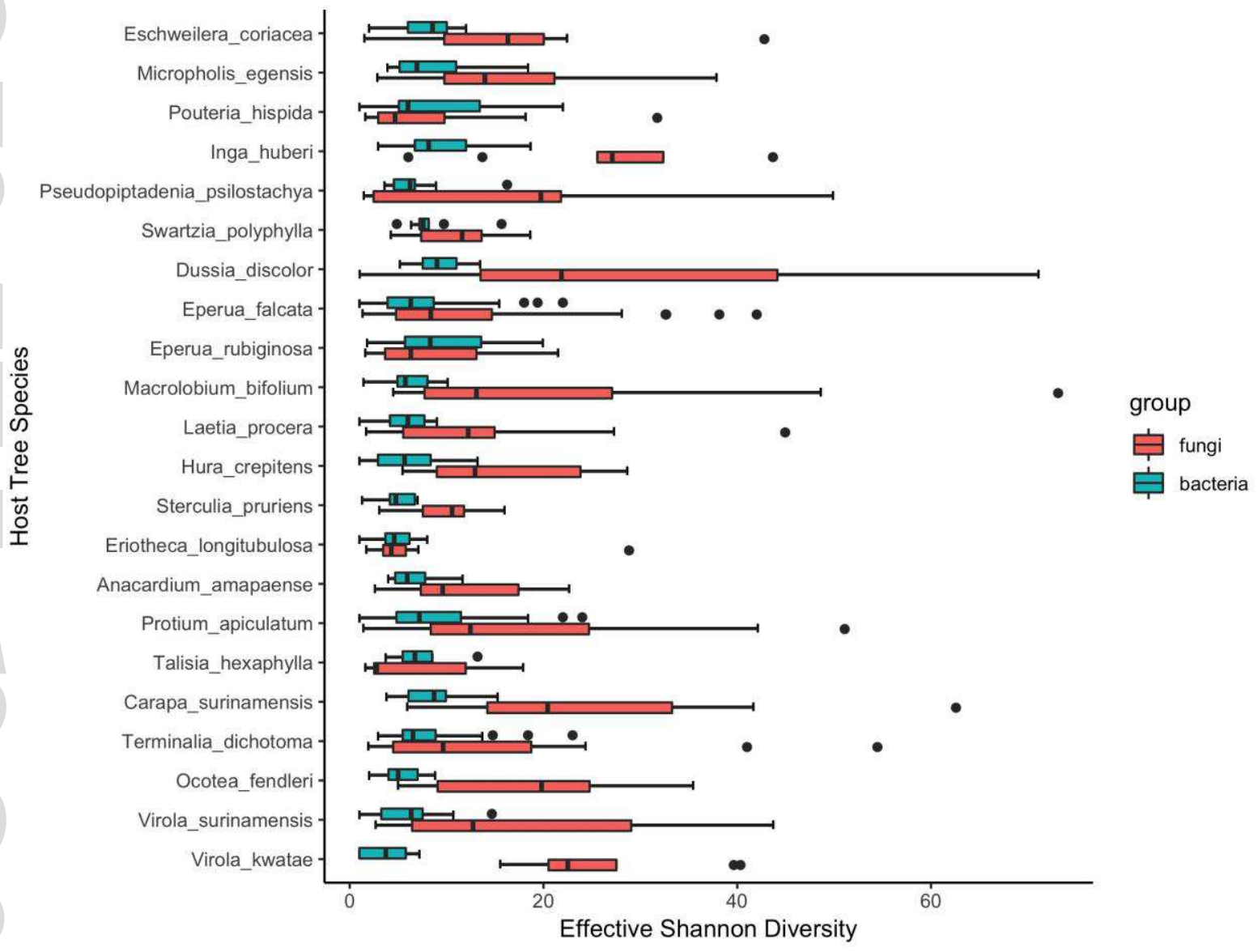

This article is protected by copyright. All rights reserved 
Figure 3: The relative occurrence of identified fungal OTUs in host trees when classed by order for a) fungi and b) bacteria. Non-identified OTUs are not included here, but represent on average $50 \%$ of all detected fungal OTUs for each sample. Host tree species are arranged by order of phylogenetic relatedness, modified from Baraloto et al. (2012). Orders are arranged in order to represent their decreasing relative occurrence from left to right, indicated by a shift from red to yellow.

a)

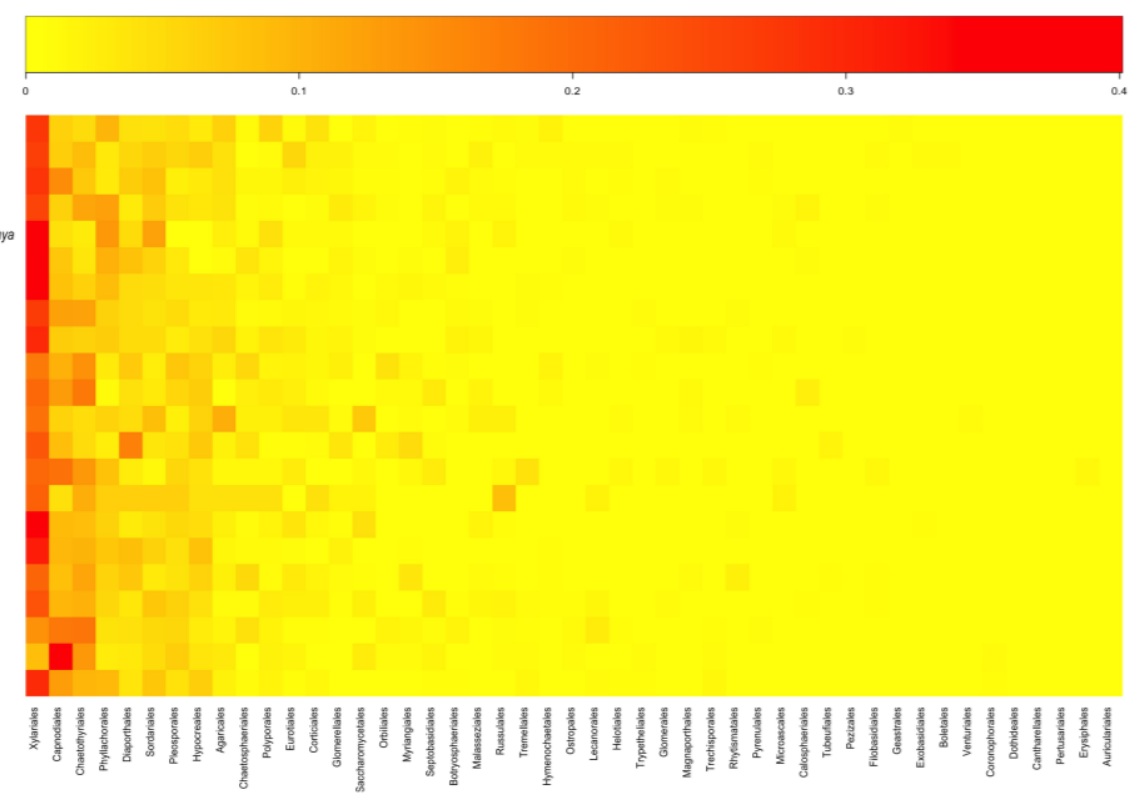

b)
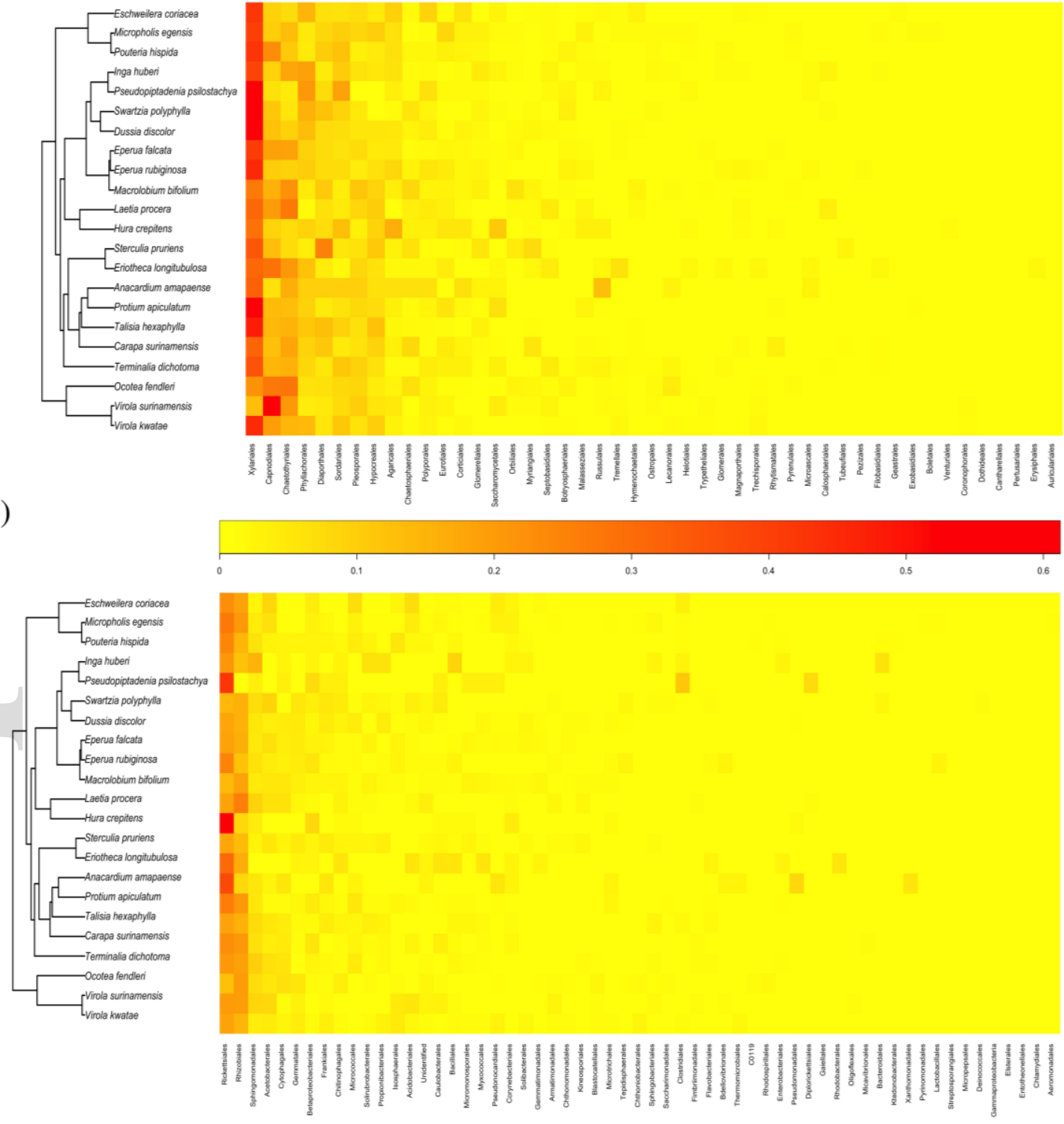

This article is protected by copyright. All rights reserved 
Figure 4: Partitioning of variance of endophyte community composition of leaves explained by host individual, host species and host branch for a) fungi and b) bacteria. Residuals indicate the amount of total variation unexplained by these factors.
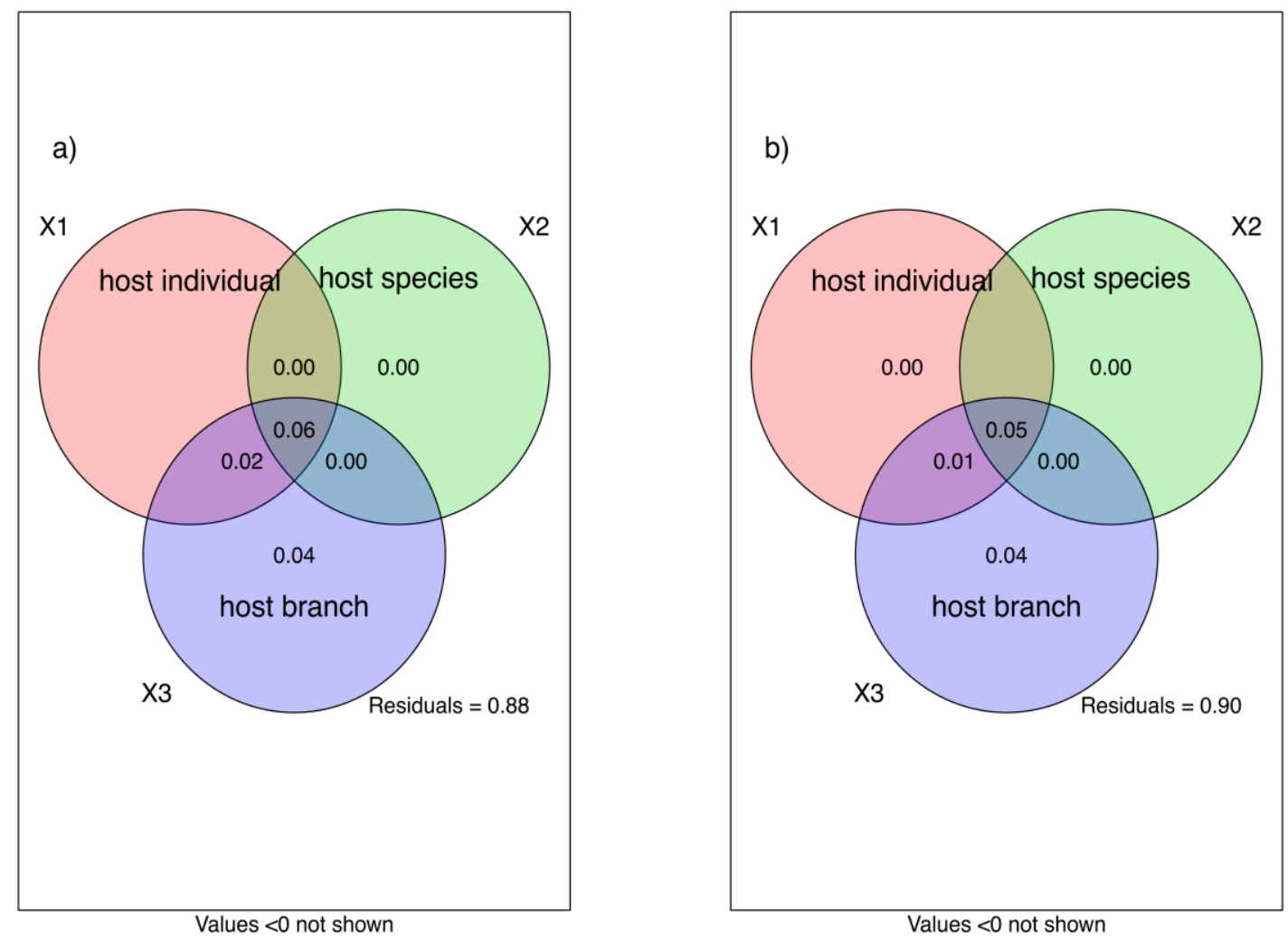

This article is protected by copyright. All rights reserved 